\title{
El sistema financiero en el Perú: Una aproximación a la banca múltiple
}

\section{INTRODUCCIÓN}

\begin{abstract}
El sistema bancario puede ser comparado con el cerebro de la economía. Asigna el capital escaso entre usos alternativos intentando orientarlo hacia donde sea más efectivo, en otras palabras, hacia donde genere los mayores rendimientos. El sistema financiero también vigila los fondos para asegurarse que son empleados en la forma comprometida.
\end{abstract}

Joseph STIGLITZ

De acuerdo con la definición establecida por la Ley General del Sistema Financiero 26702, una empresa bancaria es:

... aquella cuyo negocio principal consiste en recibir dinero del público en depósito o bajo cualquier otra modalidad contractual, y en utilizar ese dinero o su propio capital y el que obtenga de otras fuentes de financiación en conceder créditos en las diversas modalidades, o a aplicarlos a operaciones sujetas a riesgos de mercado.

Esta definición resalta una característica básica propia del sistema bancario: la intermediación; es decir, recibir fondos bajo la denominación de depósitos (ahorros, vista, plazo) con el compromiso de retribuir 
mediante un pago al agente económico que entrega estos fondos (tasa de interés pasiva) y trasladarlos a terceros, bajo la denominación de colocaciones (créditos), con la expectativa de recibir un pago por parte del agente económico que recibe los fondos (tasa de interés activa).

\section{INDICADORES DE LA BANCA MÚLTIPLE}

Al revisar algunas las cuentas de los estados financieros y otros indicadores de la banca múltiple, se puede apreciar que en los últimos cuatro años esta ha registrado un crecimiento sostenido y un proceso de fortalecimiento, los cuales se pueden explicar por el ciclo de expansión por el que atraviesan las economías nacional e internacional:

a. Balance general: En el cuadro 1 se comparan los saldos de las cuentas del balance general del 2005 versus el del 2001; se observa un crecimiento uniforme de aproximadamente $20 \%$ en las principales cuentas: créditos netos, depósitos, total activo y total pasivo.

\section{Cuadro 1 \\ Banca múltiple \\ Cuentas - Balance general}

\begin{tabular}{lccc}
\hline & 31 dic. 2001 & 31 dic. 2005 & Variación (\%) \\
\hline Créditos netos & 31.807 & 39.036 & 22,7 \\
Depósitos & 43.990 & 52.917 & 20,3 \\
Total activo & 61.812 & 75.453 & 22,1 \\
Total pasivo & 55.700 & 68.236 & 22,5 \\
Total patrimonio & 6.112 & 7.217 & 18,1 \\
\hline
\end{tabular}

Cifras expresadas en millones de soles.

Fuente: Superintendencia de Banca y Seguros (SBS).

Elaboración propia.

b. Estado de ganancias y pérdidas.- En el cuadro 2 se comparan los saldos de las cuentas del estado de ganancias y pérdidas del 2005 versus el del 2001; se pueden apreciar variaciones significativas en algunas cuentas:

- Los ingresos financieros casi se han mantenido constantes y los gastos financieros se han reducido en $40 \%$.

- El margen financiero se ha incrementado en $61 \%$ y la utilidad neta se ha incrementado en $443 \%$. 


\section{Cuadro 2}

Banca múltiple

Cuentas - Estado de ganancias y pérdidas

\begin{tabular}{lccc}
\hline & 31 dic. 2001 & 31 dic. 2005 & Variación (\%) \\
\hline Ingresos financieros & 6.058 & 6.117 & 1,0 \\
Gastos financieros & 2.898 & 1.734 & $-40,2$ \\
Margen financiero neto & 2.413 & 3.893 & 61,3 \\
Utilidad neta & 273 & 1.483 & 443,2 \\
\hline
\end{tabular}

Cifras expresadas en millones de soles.

Fuente: SBS.

Elaboración propia.

c. Indicadores diversos.- En el cuadro 3 se comparan algunos indicadores del 2005 versus los del 2001, sobre los cuales se puede comentar lo siguiente:

El indicador de la cartera atrasada [(créditos vencidos + créditos en cobranza judicial)/créditos directos] muestra una mejora notable en la calidad de la cartera, es decir, una reducción importante en la morosidad.

Los ratios de rentabilidad utilidad neta/patrimonio y utilidad neta/activo permiten apreciar una recuperación notable de los márgenes obtenidos por el sector.

\begin{tabular}{lcc} 
& $\begin{array}{c}\text { Cuadro 3 } \\
\text { Banca múltiple } \\
\text { Indicadores diversos }\end{array}$ \\
\hline & $\begin{array}{l}\text { 31 dic. 2001 } \\
\%\end{array}$ & $\begin{array}{c}\text { 31 dic. 2005 } \\
\%\end{array}$ \\
\hline Cartera atrasada/créditos directos & 9,0 & 2,1 \\
Utilidad neta/patrimonio & 4,5 & 20,5 \\
Utilidad neta/activo & 0,4 & 2,0 \\
Número de oficinas* & 822 & 841 \\
& & \\
\hline
\end{tabular}

\footnotetext{
* Al 30 de setiembre del 2005.

Fuente: SBS.

Elaboración propia.
} 


\section{CARTERA DE CRÉDITOS}

En la formación de la cartera de créditos de la banca múltiple se consideran cuatro categorías o segmentos de créditos: comerciales, de consumo, a las microempresas e hipotecarios. Los créditos comerciales corresponden a los créditos otorgados a las empresas medianas y grandes (segmento corporativo).

En el cuadro 4 se comparan los saldos de la cartera de créditos de la banca múltiple al 31 de diciembre del 2005 versus los saldos al 31 de diciembre del 2001; se observa una reducción del segmento de créditos comerciales de $6,9 \%$, en tanto que el total de créditos muestra un crecimiento de $14 \%$.

\section{Cuadro 4 \\ Banca múltiple}

\begin{tabular}{lccc}
\hline & 31 dic. 2001 & 31 dic. 2005 & Variación (\%) \\
\hline Comerciales & 28.985 & 26.971 & $-6,9$ \\
Consumo & 3.199 & 6.274 & 96,1 \\
Microempresas & 930 & 2.173 & 133,7 \\
Hipotecarios & 3.576 & 6.471 & 81,0 \\
Total créditos & 36.690 & 41.889 & 14,2 \\
\hline
\end{tabular}

Cifras expresadas en millones de soles.

Fuente: SBS.

Elaboración propia.

En los gráficos 1 y 2 se puede observar que al 31 de diciembre del 2001 los créditos comerciales representaban el $79 \%$ de la cartera de créditos directos y al 31 de diciembre del 2005 representaban el 64,4\% de esta cartera, respectivamente. Esta reducción en la participación de los créditos comerciales se explica por el crecimiento de otras categorías, como los créditos de consumo, a las microempresas e hipotecarios. Si bien la tendencia indica una reducción de la participación de los créditos comerciales sobre la cartera de créditos, el valor registrado al 31 de diciembre del 2005 indica que aún se mantiene el predominio de las colocaciones para el segmento corporativo. 


\section{Gráfico 1}

Banca múltiple

Cartera de créditos - diciembre 2001

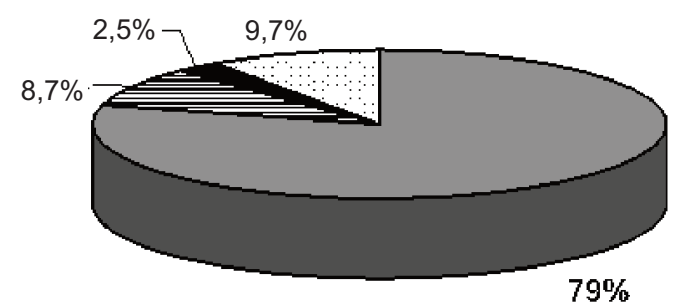

$\square$ Comerciales 目Consumo QMicroempresa $\square$ Hipotecarios

Fuente: SBS.

Elaboración propia.

Gráfico 2

Banca múltiple

Cartera de créditos - diciembre 2005

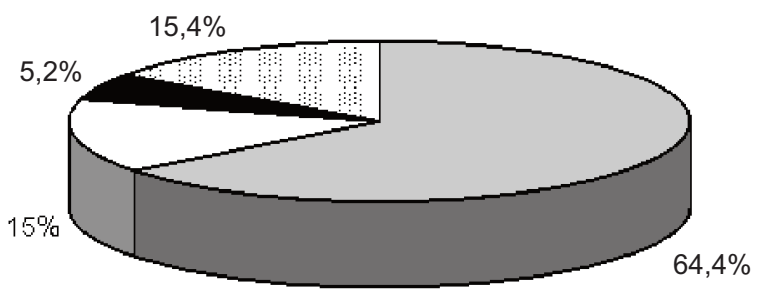

\section{$\square$ Comerciales $\square$ Consumo Dicroempresa $\square$ Hipotecarios}

Fuente: SBS.

Elaboración propia.

\section{OTROS INTERMEDIARIOS FINANCIEROS}

De acuerdo con el marco legal vigente en el sistema financiero peruano, en forma adicional a las empresas bancarias también participan otras instituciones, que a continuación detallamos.

\subsection{Cajas municipales de ahorro y crédito}

De acuerdo con la definición establecida por la Ley 26702, la Caja Municipal de Ahorro y Crédito (CMAC) "es aquella que capta recursos del 
público y cuya especialidad consiste en realizar operaciones de financiamiento, preferentemente a las pequeñas y microempresas".

El segmento de cajas municipales de ahorro y crédito está formado por 14 instituciones. A continuación se muestra la participación en el mercado de estas instituciones en créditos directos (cuadro 5).

\begin{tabular}{|c|c|c|c|}
\hline \multicolumn{4}{|c|}{$\begin{array}{c}\text { Cuadro } 5 \\
\text { Créditos directos } \\
\text { Monto (millones de soles) }\end{array}$} \\
\hline 31 dic. 2005 & Monto & $\begin{array}{c}\text { Participación } \\
(\%)\end{array}$ & $\begin{array}{l}\text { Porcentaje } \\
\text { acumulado }\end{array}$ \\
\hline CMAC Piura & 480 & 16,91 & 16,91 \\
\hline CMAC Arequipa & 465 & 16,38 & 33,30 \\
\hline CMAC Trujillo & 458 & 16,14 & 49,44 \\
\hline CMAC Sullana & 262 & 9,23 & 58,67 \\
\hline CMAC Cusco & 247 & 8,70 & 67,37 \\
\hline CMAC Huancayo & 231 & 8,14 & 75,51 \\
\hline CMCP Lima & 157 & 5,53 & 81,04 \\
\hline CMAC Tacna & 142 & 5,00 & 86,05 \\
\hline CMAC Ica & 118 & 4,16 & 90,20 \\
\hline CMAC Maynas & 89 & 3,14 & 93,34 \\
\hline CMAC Paita & 78 & 2,75 & 96,09 \\
\hline CMAC Del Santa & 74 & 2,61 & 98,70 \\
\hline CMAC Pisco & 25 & 0,88 & 99,58 \\
\hline CMAC Chincha & 12 & 0,42 & 100,00 \\
\hline & 2.838 & 100,00 & \\
\hline
\end{tabular}

Fuente: SBS.

Se debe resaltar que en el caso de Lima, la institución está constituida como una Caja Municipal de Crédito Popular (CMCP); de acuerdo con la definición establecida por la ley, una CMCP "es aquella especializada en otorgar crédito pignoraticio al público en general, encontrándose también facultada para efectuar operaciones activas y pasivas con los respectivos Concejos Provinciales y Distritales y con las empresas municipales dependientes de los primeros, así como para brindar servicios bancarios a dichos concejos y empresas". 


\subsection{Empresas de arrendamiento financiero}

De acuerdo con la definición establecida por la ley, "su especialidad consiste en la adquisición de bienes muebles e inmuebles, los que serán cedidos en uso a una persona natural o jurídica, a cambio del pago de una renta periódica y con la opción de comprar dichos bienes por un valor predeterminado".

El segmento de empresas de arrendamiento financiero está formado por seis instituciones. A continuación se muestra la participación de mercado de estas instituciones en créditos directos (cuadro 6).

\section{Cuadro 6 \\ Créditos directos \\ Monto (millones de soles)}

\begin{tabular}{llrrr}
\hline & 31 dic. 2005 & Monto & $\begin{array}{c}\text { Participación } \\
(\%)\end{array}$ & $\begin{array}{r}\text { Porcentaje } \\
\text { acumulado }\end{array}$ \\
\hline 1 & Credileasing & 843 & 51,78 & 51,78 \\
2 & América Leasing & 354 & 21,74 & 73,53 \\
3 & Wiese Leasing & 321 & 19,72 & 93,24 \\
4 & Citileasing & 72 & 4,42 & 97,67 \\
5 & Mitsui & 21 & 1,29 & 98,96 \\
6 & Leasing Total & 17 & 1,04 & 100,00 \\
\hline
\end{tabular}

Fuente: SBS.

Las tres instituciones con mayor participación en el mercado son Credileasing, América Leasing y Wiese Leasing, las cuales representan el $93 \%$ de los créditos directos, por lo que para este sector se puede afirmar que existe un elevado nivel de concentración.

\subsection{Empresas financieras}

Según la definición establecida por la ley, empresa financiera "es aquella que capta recursos del público y cuya especialidad consiste en facilitar las colocaciones de primeras emisiones de valores, operar con valores mobiliarios y brindar asesoría de carácter financiero".

El segmento de empresas financieras está formado por tres instituciones. A continuación se muestra la participación en el mercado de estas instituciones en créditos directos (cuadro 7). 


\section{Cuadro 7 \\ Créditos directos \\ Monto (millones de soles)}

\begin{tabular}{llrrr}
\hline 31 dic. 2005 & Monto & $\begin{array}{r}\text { Participación } \\
(\%)\end{array}$ & $\begin{array}{r}\text { Porcentaje } \\
\text { acumulado }\end{array}$ \\
\hline 1 & Financiera CMR & 701 & 57,13 & 57,13 \\
2 & Financiera Cordillera & 521 & 42,46 & 99,59 \\
3 & Volvo Finance & 5 & 0,41 & 100,00 \\
& & 1.227 & 100,00 & \\
\hline
\end{tabular}

Fuente: SBS.

Las dos instituciones con mayor participación en el mercado, Financiera CMR y Financiera Cordillera, representan el 99\% de los créditos directos, por lo que para este sector se puede afirmar que existe un elevado nivel de concentración.

La Financiera CMR pertenece al grupo Falabella, el cual, según la revista América Economía, registró ingresos por US\$3.854 millones durante el 2005. En el Perú Falabella maneja los negocios Saga-Falabella, Supermercados Tottus y Sodimac.

La Financiera Cordillera pertenece al grupo Ripley, el cual, según la revista América Economía, registró ingresos por US\$1.124 millones durante el 2005.

Estas empresas respaldan y potencian el desarrollo de las operaciones de sus grupos comerciales (Saga-Falabella y Ripley) y también desarrollan negocios financieros que refuerzan la generación de flujos y rentabilidad para sus respectivas casas matrices.

\subsection{Cajas rurales de ahorro y crédito}

De acuerdo con lo que establece la ley, la Caja Rural de Ahorro y Crédito (CRAC) "es aquella que capta recursos del público y cuya especialidad consiste en otorgar financiamiento preferentemente a la mediana, pequeña y microempresa del ámbito rural". 
El segmento de cajas rurales de ahorro y crédito está formado por 12 instituciones. A continuación se muestra la participación en el mercado de estas instituciones de créditos directos (cuadro 8).

\section{Cuadro 8 \\ Créditos directos \\ Monto (millones de soles)}

\begin{tabular}{llrrc}
\hline \multicolumn{1}{c}{ 31 dic. 2005 } & Monto & $\begin{array}{r}\text { Participación } \\
(\%)\end{array}$ & $\begin{array}{r}\text { Porcentaje } \\
\text { acumulado }\end{array}$ \\
\hline 1 & CRAC Nor Perú & 103 & 19,22 & 19,22 \\
2 & CRAC Señor de Luren & 95 & 17,72 & 36,94 \\
3 & CRAC Cajasur & 85 & 15,86 & 52,80 \\
4 & CRAC San Martín & 74 & 13,81 & 66,60 \\
5 & CRAC Credinka & 43 & 8,02 & 74,63 \\
6 & CRAC Profinanzas & 26 & 4,85 & 79,48 \\
7 & CRAC Cruz de Chalpón & 23 & 4,29 & 83,77 \\
8 & CRAC Prymera & 22 & 4,10 & 87,87 \\
9 & CRAC Cajamarca & 20 & 3,73 & 91,60 \\
10 & CRAC Libertadores de Ayacucho & 18 & 3,36 & 94,96 \\
11 & CRAC Chavín & 14 & 2,61 & 97,57 \\
12 & CRAC Los Andes & 13 & 2,43 & 100,00 \\
& & 536 & 100,00 & \\
\hline
\end{tabular}

Fuente: SBS.

Las cuatro instituciones con mayor participación en el mercado, CRAC Nor Perú, CRAC Señor de Luren, CRAC CajaSur y CRAC San Martín, representan el 66\% de los créditos directos, por lo que para este sector se puede afirmar que existe un elevado nivel de concentración.

\subsection{Empresas de desarrollo de la pequeña y microempresa}

De acuerdo con la definición establecida por la ley, la empresa de desarrollo de la pequeña y microempresa (Edpyme) "es aquella cuya especialidad consiste en otorgar financiamiento preferentemente a los empresarios de la pequeña y microempresa”.

El segmento de las Edpyme está formado por 14 instituciones. A continuación se muestra la participación en el mercado de estas instituciones en créditos directos (cuadro 9). 


\section{Cuadro 9 \\ Créditos directos \\ Monto (millones de soles)}

\begin{tabular}{llrrc}
\hline 31 dic. 2005 & Monto & $\begin{array}{r}\text { Participación } \\
(\%)\end{array}$ & $\begin{array}{r}\text { Porcentaje } \\
\text { acumulado }\end{array}$ \\
\hline 1 & Edpyme Edyficar & 188 & 34,94 & 34,94 \\
2 & Edpyme Raíz & 78 & 14,50 & 49,44 \\
3 & Edpyme Confianza & 76 & 14,13 & 63,57 \\
4 & Edpyme Crear Arequipa & 57 & 10,59 & 74,16 \\
5 & Edpyme Proempresa & 40 & 7,43 & 81,60 \\
6 & Edpyme Crear Tacna & 30 & 5,58 & 87,17 \\
7 & Edpyme Nueva Visión & 19 & 3,53 & 90,71 \\
8 & Edpyme Efectiva & 13 & 2,42 & 93,12 \\
9 & Edpyme Alternativa & 8 & 1,49 & 94,61 \\
10 & Edpyme Credivisión & 7 & 1,30 & 95,91 \\
11 & Edpyme Crear Trujillo & 7 & 1,30 & 97,21 \\
12 & Edpyme Crear Cusco & 7 & 1,30 & 98,51 \\
13 & Edpyme Pro Negocios & 5 & 0,93 & 99,44 \\
14 & Edpyme Solidaridad & 3 & 0,56 & 100,00 \\
\hline
\end{tabular}

Fuente: SBS.

Las cuatro instituciones con mayor participación en el mercado son las Edpyme Edyficar, Raíz, Confianza y Crear Arequipa, que representan el $74 \%$ de los créditos directos, por lo que para este sector se puede afirmar que existe un elevado nivel de concentración.

El caso del segmento de las microfinanzas, en el cual trabajan las Edpyme, resulta particularmente relevante para la realidad de un país como el Perú; en nuestro país, según estimados del Banco Interamericano de Desarrollo, la microempresa genera empleo para más del $70 \%$ de la población económicamente activa.

La Edpyme Edyficar, que es la institución de este segmento más importante, fue constituida en 1997; su principal accionista es Care Perú, que registra una participación en su accionariado del 68,5\%. Esta institución, que forma parte del grupo Care International, plantea como uno de sus objetivos básicos "incidir en las causas de la pobreza, inequidad y exclusión a través de la acción integrada en procesos de desarrollo social, económico y político, a través de tres objetivos estratégicos: desarrollo de capital humano y social, desarrollo económico sostenible y fortalecimiento de la institucionalidad democrática" (Care Perú, 2005).

De acuerdo con Mark Malloch Brown (2005), administrador del Programa de las Naciones Unidas para el Desarrollo (PNUD), "las microfi- 
nanzas permiten apoderar a la gente pobre, en particular a las mujeres, por lo que se han convertido en un instrumento clave para alcanzar los 'Objetivos de Desarrollo del Milenio', establecidos por las Naciones Unidas, en particular el primer objetivo: reducir a la mitad, entre $1990 \mathrm{y}$ 2015, el porcentaje de personas cuyos ingresos sean inferiores a 1 dólar por día y el porcentaje de personas que padecen hambre”.

\section{BANCA MÚLTIPLE: ANÁLISIS DEL SECTOR}

A continuación se presenta el análisis de algunas características asociadas con la banca múltiple.

\subsection{Concentración}

Si la participación de mercado de las cuatro empresas más grandes excede el 60\%, esto se considera como indicador de un mercado que está altamente concentrado y que, al ser dominado por unas pocas empresas, coincide con la definición de oligopolio.

Michel PARKin y Gerardo Esquivel

La banca múltiple está formada por 12 instituciones financieras intermediarias. A continuación se muestra la participación en el mercado de estas instituciones en créditos directos (cuadro 10) y en depósitos totales (cuadro 11).

\section{Cuadro 10 \\ Créditos directos \\ Monto (millones de soles)}

\begin{tabular}{llrrr}
\hline \multicolumn{1}{c}{ 31 dic. 2005 } & Monto & $\begin{array}{r}\text { Participación } \\
(\%)\end{array}$ & $\begin{array}{r}\text { Porcentaje } \\
\text { acumulado }\end{array}$ \\
\hline 1 Banco de Crédito del Perú* & 13.424 & 32,05 & 32,05 \\
2 & BBVA Banco Continental & 9.607 & 22,93 & 54,98 \\
3 & Banco Wiese Sudameris & 6.235 & 14,88 & 69,87 \\
4 & Interbank $^{*}$ Citibank & 4.154 & 9,92 & 79,78 \\
6 & Banco Interamericano de Finanzas & 1.690 & 4,03 & 83,82 \\
7 & Banco Sudamericano* & 1.660 & 3,96 & 87,78 \\
8 & Banco Financiero & 1.593 & 3,80 & 91,58 \\
9 & Banco del Trabajo & 1.307 & 3,12 & 94,70 \\
10 Mibanco & 1.079 & 2,58 & 97,28 \\
11 Banco de Comercio & 709 & 1,69 & 98,97 \\
12 BNP Paribas Andes & 426 & 1,02 & 99,99 \\
Total & 4 & 0,01 & 100,00 \\
\hline
\end{tabular}

Fuente: SBS. 


\section{Cuadro 11 \\ Depósitos totales \\ Monto (millones de soles)}

\begin{tabular}{llrrr}
\hline \multicolumn{1}{c}{ 31 dic. 2005 } & Monto & $\begin{array}{r}\text { Participación } \\
(\%)\end{array}$ & $\begin{array}{r}\text { Porcentaje } \\
\text { acumulado } \\
(\%)\end{array}$ \\
\hline 1 & & & 34,35 & 34,35 \\
2 & Banco de Crédito del Perú* & 18.179 & 27,44 & 61,79 \\
3 & BBVA Banco Continental & 14.520 & 15,86 & 77,66 \\
4 & Banco Wiese Sudameris & 8.393 & 8,54 & 86,20 \\
5 & Interbank & 4.521 & 8,54 & 89,38 \\
6 & Banco Interamericano de Finanzas & 1.682 & 3,18 & 91,87 \\
7 & Banco Financiero & 1.321 & 2,50 & 94,19 \\
8 & Citibank & 1.225 & 2,31 & 96,45 \\
9 & Banco del Trabajo & 1.194 & 2,26 & 97,99 \\
10 & Mibanco & 815 & 1,54 & 99,00 \\
11 & Banco de Comercio & 537 & 1,01 & 99,93 \\
12 & BNP Paribas Andes & 493 & 0,93 & 100,00 \\
& Total & 36 & 0,07 & \\
\hline
\end{tabular}

* No se consideran las sucursales en el exterior.

Se consideran: Obligaciones con el público y depósitos del sistema financiero. Fuente: SBS.

Las cuatro instituciones con mayor participación en el mercado son el Banco de Crédito, el BBVA Banco Continental, el Banco Wiese Sudameris e Interbank, los cuales representan el $80 \%$ de los créditos directos, el $86 \%$ de los depósitos y el $83 \%$ del total de activos de la banca múltiple. Estos volúmenes de participación en el mercado plantean un nivel de concentración relevante para el sector.

La característica de la concentración también se puede observar en el negocio bancario a escala mundial. Según la revista The Banker los 25 bancos más grandes representan el 39\% del total de activos de la lista de los 1.000 bancos más grandes del mundo (The Banker, 2005). 


\subsection{Bancarización o profundidad}

La crisis del capitalismo, fuera de los paises avanzados no se debe a que la globalización internacional esté fracasando sino a que los países en vías de desarrollo... no han podido "globalizar" el capital en su territorio. La mayor parte de las personas en estos países ve al capitalismo como un club privado, un sistema discriminatorio que sólo beneficia a Occidente y a las élites que viven dentro de las campanas de vidrio instaladas en los países pobres.

HERNANDo DE Soto

La bancarización o profundidad del sistema bancario se entiende como la relevancia que tiene la intermediación financiera con respecto al valor de los bienes y servicios producidos por la economía de un país. Este indicador puede ser calculado dividiendo el valor del total de créditos directos del sistema bancario entre el valor del Producto Interno Bruto (PIB).

Los créditos totales correspondientes al sistema bancario en el Perú representan el $22 \%$ del PIB aproximadamente, siendo el promedio en América Latina de 25\% y el del grupo de países más desarrollados (G-7) igual al 80\% (BID, 2005).

De otro lado, para evaluar la profundidad del sistema bancario también hay que tomar en cuenta el tema relacionado con los costos de transacción. Una institución financiera incurre en costos administrativos relativamente bajos cuando coloca un crédito a un cliente del segmento corporativo; si el mismo importe que entrega a este cliente corporativo lo subdividiera y lo otorgara a varios clientes pertenecientes a los segmentos de pequeñas empresas o de créditos de consumo, la institución financiera incurrirá en mayores costos administrativos.

En el cuadro 12 se compara el número total de deudores de la banca múltiple al 31 de diciembre del 2005 versus el número de deudores al 31 de diciembre del 2000; se observa un crecimiento significativo de $91 \%$ en el número de deudores. Sin embargo, si se tienen en cuenta otras variables, como la población económicamente activa del Perú, la que supera los 12 millones de personas, se puede afirmar que el acceso al crédito en el Perú es un proceso complejo y que existe la necesidad de incorporar una mayor cantidad de clientes nuevos al sistema. 


\section{Cuadro 12 \\ Banca múltiple}

\begin{tabular}{lccc}
\hline & 31 dic. 2000 & 31 dic. 2005 & $\begin{array}{c}\text { Variación } \\
\%\end{array}$ \\
\hline Número total de deudores & 1.192 .469 & 2.277 .725 & 91,0 \\
\hline
\end{tabular}

Fuente: SBS.

Elaboración propia.

Según los datos de una revista especializada, de un universo de 2.500.000 contribuyentes registrados y activos en la Sunat, un pequeño grupo de 261 contribuyentes explican el 55\% de la recaudación (Procapitales, 2003); este tema debe ser considerado cuando se trata de explicar la característica de la profundidad del sistema bancario.

\subsection{Riesgo}

Una crisis puede desencadenar un círculo vicioso por el cual los bancos recortan su financiamiento, lo que lleva a las empresas a recortar su actividad, lo que a su vez reduce la producción y las rentas.

Joseph STIGLitz

Las estadísticas del Banco Mundial revelan que en el 2004 el PIB del Perú representó el 0,16\% del PIB mundial (Word Bank Group, 2004). Para el 2004 y para el periodo enero-junio del 2005, el rubro de productos mineros representó el $54 \%$ del total de exportaciones peruanas (Proinversión). Así, la economía peruana es una economía pequeña, dependiente de los precios de los commodities, los cuales se fijan en los mercados internacionales; adicionalmente, el Perú forma parte de una región caracterizada por el desarrollo de procesos políticos con horizontes de tiempo bastante cortos. Los fundamentos explicados están asociados a los niveles de riesgo de mercado del país.

De acuerdo con un estudio de Grade, "el tamaño del sector informal en el Perú se ha calculado dentro de un rango que varía entre el 40 y $60 \%$ del PBI" (Grade, 2004); este hecho eleva el nivel de dificultad para realizar una evaluación crediticia estándar. La informalidad de los negocios se asocia directamente al riesgo de crédito. 
Ambos, el riesgo de mercado y el riesgo de crédito, tienen un impacto negativo sobre el acceso al crédito y los niveles de las tasas de interés.

\subsection{Tasas de interés}

En los siguientes cuadros se presentan datos relacionados con el spread absoluto de las tasas de interés promedio (tasa de interés activa-tasa de interés pasiva), en moneda nacional (cuadro 13) y en moneda extranjera (cuadro 14).

Cuadro 13

\begin{tabular}{lcc}
\hline & $\begin{array}{c}31 \text { dic. 2002 } \\
\%\end{array}$ & $\begin{array}{c}31 \text { dic. 2005 } \\
\%\end{array}$ \\
\hline Tasa activa promedio anual (TAMN) & 20,70 & 23,60 \\
Tasa pasiva promedio anual (TPMN) & 3,60 & 2,60 \\
Spread absoluto & 17,10 & 21,00 \\
\hline
\end{tabular}

Fuente:Banco Central de Reserva del Perú (BCRP).

Elaboración propia.

\section{Cuadro 14}

\begin{tabular}{lcc}
\hline & $\begin{array}{c}31 \text { dic. 2002 } \\
\%\end{array}$ & $\begin{array}{c}31 \text { dic. 2005 } \\
\%\end{array}$ \\
\hline Tasa activa promedio anual (TAME) & 10,20 & 10,40 \\
Tasa pasiva promedio anual (TPME) & 1,30 & 1,80 \\
Spread absoluto & 8,90 & 8,60 \\
\hline
\end{tabular}

Fuente:Banco Central de Reserva del Perú (BCRP).

Elaboración propia.

\subsection{Presencia de la banca extranjera}

Durante los últimos años, las cuatro principales instituciones del sistema han registrado cambios en su composición accionaria:

- Banco de Crédito.- Es una subsidiaria del holding Credicorp Ltd. Este holding tiene una participación de 96,2\% en el capital del banco. Las Administradoras de Fondos de Pensiones (AFP) en conjunto cuentan con una participación de $40 \%$ en el capital de Credicorp. 
- BBVA Banco Continental.- En 1995 se realiza la privatización del banco. Hoy es una subsidiaria del holding Continental S.A. Este holding tiene una participación de 92\% en el capital del banco. El Banco Bilbao Vizcaya Argentaria (BBVA) de España y el grupo Brescia tienen una participación de 50\% cada uno en el capital del holding Continental.

- Banco Wiese Sudameris.- El año 1999 el Grupo Wiese vende su participación a Banca Intesa de Italia. En diciembre del 2005 el Scotia Bank, banco canadiense, anuncia la adquisición del 82\% del capital del banco.

- Interbank.- En 1994 se realiza la privatización del banco. Hoy es una subsidiaria del holding IFH Perú Ltd. Este holding tiene una participación de 83\% en el capital del banco; destaca la participación del grupo Rodríguez-Pastor.

La banca múltiple está formada por 12 instituciones y en ocho de ellas la banca extranjera cuenta con una participación relevante: BBVA Banco Continental, Banco Wiese Sudameris, Banco Interamericano de Finanzas (BIF), Banco Sudamericano, Citibank, BNP Paribas, Banco Financiero y Banco del Trabajo. Al 31 de diciembre del 2005, estas ocho instituciones explican el 55\% del total de créditos directos y también el 55\% del total de depósitos de la banca múltiple.

\section{REFLEXIONES FINALES}

La banca múltiple es un sector muy relevante para el sistema económico y de su estabilidad y solidez dependen variables económicas como la producción, el empleo y el nivel de precios.

Este sector ha superado con éxito la crisis financiera internacional ocurrida entre 1998 y el 2001. A la fecha se ha consolidado como un negocio sólido, rentable y con un nivel de supervisión reconocido internacionalmente. La participación mayoritaria de la banca extranjera en el sector confirma que el negocio es atractivo; asimismo, los fundamentos económicos del país permitieron una performance positiva del sector durante el 2006.

Temas como incrementar la competencia y la bancarización o reducir el nivel del spread financiero constituyen retos complejos para las propias instituciones financieras, pero también para los grupos de in- 
terés relacionados: el Estado, los sectores no bancarizados, las organizaciones y foros especializados.

Se requiere de un trabajo conjunto y complementario entre la banca múltiple y el Estado para diseñar planes y estrategias que contribuyan a lograr nuevos y mayores avances en temas críticos para el país, como la inclusión en el sistema financiero de mayores segmentos de la población, el crecimiento económico y la reducción de la pobreza.

\section{BIBLIOGRAFÍA}

Banco Central de Reserva del Perú. Memoria Anual BCRP, años 2002 y 2005. Lima: BCRP.

Banco Interamericano de Desarrollo. Progreso económico y social de América Latina. Informe. 2005.

Care Perú. Objetivos estratégicos. Memoria institucional 2005. Lima: Care Perú.

De Soto, Hernando. El misterio del capital. Lima: Empresa Editora El Comercio, 2000.

Grade. Evasión tributaria e informalidad en el Perú. Lima, 2004.

Mallock Brown, Mark. Programa de las Naciones Unidas para el Desarrollo (PNUD). Informe anual del administrador. Junio 2005.

Parkin, Michael y Gerardo Esquivel. Microeconomía. México: Pearson Educación, 2001.

Procapitales 68. Lima, octubre del 2003.

Proinversión. Guía de inversiones en el Perú. Lima: Proinversión, 2005.

Stiglitz, Joseph. El malestar en la globalización. Madrid: Santillana, 2003.

Superintendencia de Banca, Seguros y AFP. Memoria Anual. Años 2000, 2001, 2002 y 2005.

The Banker. "The top one thousand world banks". Julio del 2005.

World Bank Group. Data \& Statistics. Total GDP, 2004. 